\title{
El médico ante los tribunales: análisis de las sentencias judiciales relacionadas con la responsabilidad profesional médica en España.
}

\author{
Perea Pérez, Bernardo, Santiago Sáez, Andrés, Labajo \\ González, Elena, Albarrán Juan, María Elena, Dorado \\ Fernández, Enrique y García Martín, Ángel.
}

Cita:

Perea Pérez, Bernardo, Santiago Sáez, Andrés, Labajo González, Elena, Albarrán Juan, María Elena, Dorado Fernández, Enrique y García Martín, Ángel (2013). El médico ante los tribunales: análisis de las sentencias judiciales relacionadas con la responsabilidad profesional médica en España. Revista Española de Medicina Legal, 39 (4), 130-134.

Dirección estable: https://www.aacademica.org/elenalabajogonzalez/15 ARK: https://n2t.net/ark:/13683/pcQr/4SS

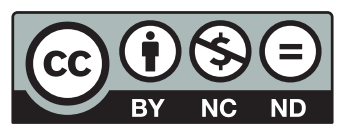




\title{
El médico ante los tribunales: análisis de las sentencias judiciales relacionadas con la responsabilidad profesional médica en España
}

\author{
Bernardo Perea-Pérez ${ }^{a}$, Andrés Santiago-Saéz ${ }^{\mathrm{a}, \mathrm{b}}$, María Elena Labajo-González ${ }^{\mathrm{a}, *}$, \\ María Elena Albarrán-Juan ${ }^{a}$, Enrique Dorado-Fernández ${ }^{a}$ y Ángel García-Martín ${ }^{c}$
}

\author{
a Escuela Medicina Legal y Forense de Madrid, Facultad de Medicina, Universidad Complutense de Madrid, Madrid, España \\ b Servicio de Medicina Legal, Hospital Clínico San Carlos, Madrid, España \\ c Facultativo Madrid Salud, Ayuntamiento de Madrid, Madrid, España
}

Recibido el 29 de abril de 2013; aceptado el 29 de mayo de 2013

Disponible en Internet el 18 de julio de 2013

\section{PALABRAS CLAVE \\ Responsabilidad; \\ Negligencia; \\ Condenas; \\ Cuantía \\ indemnizatoria; \\ Especialidad médica}

\begin{abstract}
Resumen
Introducción: Existen pocos datos objetivos referentes al número de reclamaciones legales contra médicos, de su distribución por especialidades o de sus consecuencias (porcentaje de condenas o cuantía de las indemnizaciones).

Material y método: Se han analizado todas las sentencias presentes en las bases de datos jurídicas (CENDOJ y Westlaw-Aranzadi) hasta el año 2010. Se seleccionaron 2.817 referidas a praxis médica y que contuviesen datos completos.

Resultados: La mayoría de las sentencias, el 65,2\% (1.837) se dictaron en el período 2001-2010, procediendo la mayoría de la jurisdicción civil (85\%). Las especialidades más reclamadas judicialmente fueron ginecología y obstetricia $(13,5 \%)$, cirugía ortopédica y traumatología $(12,3 \%)$, urgencias $(9,7 \%)$, cirugía y medicina estética $(8,1 \%)$ y enfermedades infecciosas $(6 \%)$. El $46 \%$ de las sentencias apreciaron algún tipo de mala praxis por parte del médico, y la cuantía media de las indemnizaciones fue de $83.457,5 €$.

Conclusiones: El número de sentencias motivadas por reclamaciones de mala praxis médica ha aumentado progresivamente durante el período contemplado. Las especialidades médicas más reclamadas fueron ginecología y obstetricia, y cirugía ortopédica y traumatología. Estos datos coinciden con la gran mayoría de las series similares disponibles. El porcentaje de sentencias que aprecia algún tipo de mala praxis médica (46\%) es más alto que en otras series, y la cuantía media en las indemnizaciones es 2 veces y media menor que en las series de referencia norteamericanas.

(c) 2013 Asociación Nacional de Médicos Forenses. Publicado por Elsevier España, S.L. Todos los derechos reservados.
\end{abstract}

\footnotetext{
* Autor para correspondencia.

Correo electrónico: elabajo@med.ucm.es (M.E. Labajo-González).
} 


\section{KEYWORDS}

Liability; Negligence; Convictions; Amount for damages; Medical specialty
Doctors before the courts: Analysis of court rulings related to medical professional liability in Spain

\begin{abstract}
Background: There are few objective data on the number of legal claims against doctors, their distribution by specialty, or their consequences (conviction rate or amount of compensation). Material and method: We analyzed all rulings present in legal databases (CENDOJ and WestlawAranzadi) until 2010. The 2.817 were selected regarding medical practice and that would contain complete data.

Results: Most of sentences 65.2\% (1.837) were issued in the period 2001-2010, mostly originating from civil jurisdiction (85\%). The most court claimed specialties were obstetrics and gynecology (13.5\%), orthopedic surgery and traumatology (12.3\%), emergency department $(9.7 \%)$, surgery and aesthetic medicine (8.1\%), and infectious diseases $(6 \%)$. The $46 \%$ of the judgments noted some kind of malpractice by the physician, and the average amount of compensation was $€$ 83457.5.

Conclusions: The number of judgments caused by medical malpractice claims has increased steadily during the period covered. The most claimed medical specialties were obstetrics and gynecology and orthopedic surgery. These data are consistent with the majority of similar series available. The percentage of sentences where some sort of medical malpractice is noted (46\%) is higher than in other series, and the average amount of compensation was two and 2.5 times lower than in the American reference series.

(c) 2013 Asociación Nacional de Médicos Forenses. Published by Elsevier España, S.L. All rights reserved.
\end{abstract}

\section{Introducción}

En los últimos años hemos asistido a un incremento de la presión legal de los pacientes contra médicos y otros profesionales sanitarios. Sin embargo, este hecho incuestionable no está respaldado en nuestro país por estudios que manejen datos objetivos, homogéneos y comparables. Esta falta de estudios se debe a la gran dispersión de los datos referentes a reclamaciones legales motivadas por supuesta mala praxis médica y la protección de la que gozan estos datos ${ }^{1,2}$.

La gran dispersión de datos viene dada por la gran cantidad de instancias judiciales y no judiciales ante las que se puede reclamar a un médico por su actividad profesional: departamentos de atención al paciente de hospitales y áreas de salud, oficinas del defensor del paciente de las diferentes comunidades autónomas, oficinas municipales de información al consumidor (especialmente en caso de práctica privada), comisiones deontológicas de los colegios profesionales, compañías aseguradoras de la responsabilidad civil profesional médica y juzgados (penales, civiles, contencioso-administrativos, etc.). Además, un buen número de estas reclamaciones se presentan simultánea o sucesivamente en más de una de las instituciones anteriormente mencionadas.

Existen 2 dificultades añadidas para acceder a este tipo de datos. La primera es la protección de la que gozan los ficheros que contienen datos de salud, según la Ley Orgánica 15/1999 de protección de datos de carácter personal. La segunda es que no es habitual que las instituciones depositarias compartan estos datos, recelosas de que pueda ser comparada su calidad asistencial con otras instituciones análogas (en el caso de las instituciones sanitarias) o incluso por motivaciones económicas (en el caso de las compañías aseguradoras de la responsabilidad civil médica). Aun así, existen algunos estudios como los de Perea-Pérez et al. ${ }^{1}$ (2009) o Arimany-Manso et al. ${ }^{2}$ (2013) que estudian reclamaciones sobre responsabilidad profesional en la asistencia sanitaria interpuestas en España.

Las escasas bases de datos comparables (Willis, Tirant lo Blanch, Westlaw y del Centro de Documentación Judicial [CENDOJ]) muestran un aumento en el número de reclamaciones ${ }^{3-6}$, y una distribución poco homogénea entre las diferentes especialidades médicas ${ }^{1-3,7}$. El aumento de la presión legal está teniendo repercusiones importantes en la práctica de la medicina ${ }^{8,9}$. Por un lado, los costes directos e indirectos de estas reclamaciones están aumentando de forma importante ${ }^{10-13}$. Y por otro, los médicos afectados varían su perspectiva sobre la asistencia clínica y su forma de trabajo ${ }^{14}$.

El objetivo de este trabajo es establecer una base de datos homogénea sobre las reclamaciones legales de los pacientes partiendo de las bases de datos de sentencias judiciales, y realizar un análisis descriptivo y comparativo de los resultados.

\section{Material y método}

Este estudio se ha realizado partiendo de 2.817 sentencias judiciales de los tribunales españoles de segunda - tercera instancia correspondientes a los ámbitos jurisdiccionales penal (audiencias provinciales y Tribunal Supremo), civil (audiencias provinciales y Tribunal Supremo) y contencioso-administrativo (tribunales superiores de justicia y Tribunal Supremo). Se han estudiado todas las sentencias encontradas hasta el año 2010 incluido. Estas fechas se refieren a la publicación de la sentencia. 


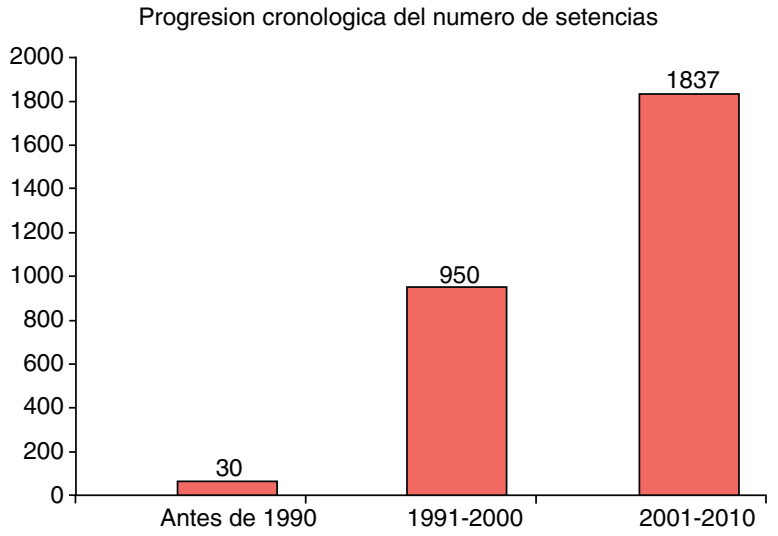

Figura 1 Evolución del número de sentencias (N. ${ }^{\circ}$ de sentencia/Período cronológico).

Las sentencias se han localizado mediante la base de datos del «Buscador del sistema de jurisprudencia» del CENDOJ, órgano técnico del Consejo General del Poder Judicial, y de la base de datos «Westlaw-Aranzadi».

La primera búsqueda dio como resultado un total de 8.997 sentencias que trataban de una u otra forma aspectos relativos a la asistencia médica. Esas 8.997 sentencias fueron revisadas $y$, debido a que se trata de textos jurídicos, los autores han utilizado unos criterios de inclusión basados en que en la sentencia figure una «supuesta» incorrecta práctica asistencial, y que esta contuviese datos claros sobre la maniobra médica cuestionada, los sanitarios implicados, las consecuencias para el paciente y sus secuelas.

Al aplicar estos criterios de inclusión, las sentencias quedaron reducidas a 2.817. El resto eran sentencias incompletas o, en su mayoría, se referían a asuntos en los que la asistencia sanitaria era secundaria y no valorada en la sentencia (accidentes de tráfico, prestaciones sanitarias, agresiones, etc.).

De cada sentencia se elaboró una ficha de datos que recogía aspectos sobre: año de publicación de la sentencia, tribunal, jurisdicción, especialidad médica reclamada, fallo de la sentencia (condena o estimación de la demanda total o parcial; o no) e indemnización económica fijada en la sentencia (en caso de condena o estimación de la demanda).

El estudio estadístico se dividió en 2 fases: una primera, donde los datos fueron registrados, y una segunda fase con un estudio descriptivo de todas las variables analizadas incluyendo el cálculo de la media en la variable cuantitativa del coste de las indemnizaciones.

\section{Resultados}

Las variables administrativas y judiciales de la muestra estudiada de las 2.817 sentencias seleccionadas se recogen en la tabla 1.

En relación al año en el que se dictaron las sentencias, y dada la gran variabilidad existente entre el momento de producción de los hechos que dieron lugar a la reclamación legal y la fecha de la sentencia, se ha optado por considerar 3 grandes períodos (fig. 1):antes de 1990 (30 sentencias), 1991-2000 (950 sentencias) y 2001-2010 (1.837 sentencias).
Tabla 1 Variables administrativas y judiciales de la muestra estudiada. Datos presentados como $n$ (\%) de 2.817 sentencias estudiadas

\begin{tabular}{lc}
\hline Variables administrativas & \\
Fecha de la sentencia & 30 \\
Antes de 1990 & 950 \\
$1991-2000$ & 1.837 \\
$2001-2010$ & \\
Tipo de tribunal & $2.106(74,7)$ \\
Audiencia Provincial & $363(12,8)$ \\
Tribunales Superiores de Justicia & $388(12,3)$ \\
Tribunal Supremo & \\
Jurisdicción & $2.395(85)$ \\
Jurisdicción civil & $239(8)$ \\
Jurisdicción penal & $183(7)$ \\
Jurisdicción contencioso-administrativa & \\
Variables judiciales & \\
Especialidad & \\
Ginecología y obstetricia & $380(13,5)$ \\
Traumatología y cirugía ortopédica & $347(12,3)$ \\
Urgencias & $271(9,7)$ \\
Cirugía y medicina estéticas & $227(8,1)$ \\
Enfermedades infecciosas & $167(6)$ \\
Odontoestomatología & $143(5)$ \\
Cirugía general y digestiva & $129(4,6)$ \\
Oftalmología & $117(4,2)$ \\
Anestesiología y reanimación & $88(3,1)$ \\
Urología & $85(3)$ \\
Resultado de la sentencia & \\
Sentencias absolutorias & \\
Sentencias condenatorias o con & $1.523(54)$ \\
estimación parcial o total de la demanda & $1.294(46)$ \\
Cuantía indemnizatoria media & \\
Especialidades médicas & \\
Especialidades quirúrgicas & $870.076 €$ \\
Urgencias & \\
UCl & \\
\hline & \\
& \\
& \\
& \\
&
\end{tabular}

En lo referente a los tribunales que dictaron las sentencias, los resultados son:

- Audiencias Provinciales: 2.106 sentencias (74,7\%).

- Tribunales Superiores de Justicia: 363 sentencias (12,8\%).

- Tribunal Supremo: 348 sentencias (12,3\%).

Respecto a la jurisdicción en la que se juzgaron las reclamaciones por mala praxis médica, su distribución es la siguiente:

- Jurisdicción civil: 2.395 sentencias (85\%).

- Jurisdicción penal: 239 sentencias (8\%).

- Jurisdicción contencioso-administrativa: 183 casos (7\%).

Las 10 especialidades médicas más reclamadas en nuestra serie se distribuyen por orden de frecuencia del siguiente modo (fig. 2):

- Ginecología y obstetricia (GO): 380 sentencias $(13,5 \%)$. 


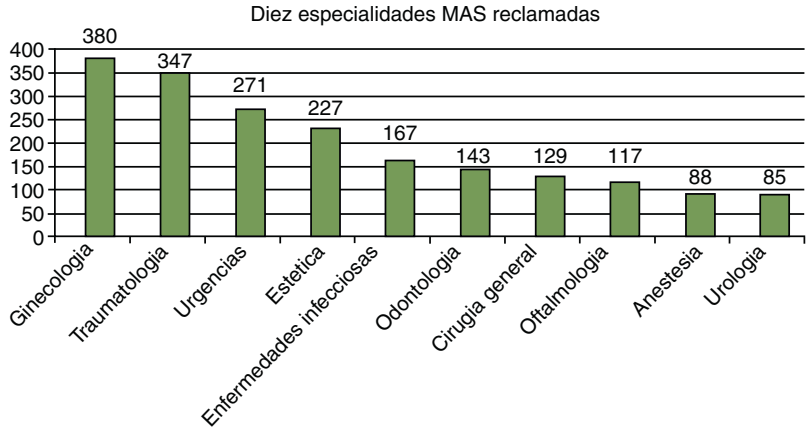

Figura 2 Especialidades médicas más reclamadas $\left(\mathrm{N} .^{\circ}\right.$ de reclamaciones/Especialidad).

- Cirugía ortopédica y traumatología (COT): 347 sentencias $(12,3 \%)$.

- Urgencias: 271 sentencias (9,7\%). Es posible que la práctica asistencial en los servicios de urgencias contemplen procedimientos y maniobras correspondientes a múltiples especialidades.

- Cirugía y medicina estéticas: 227 sentencias (8,1\%).

- Enfermedades infecciosas: 167 sentencias (6\%).

- Odontoestomatología: 143 sentencias (5\%).

- Cirugía general y digestiva: 129 sentencias (4,6\%).

- Oftalmología: 117 sentencias (4,2\%).

- Anestesiología y reanimación: 88 sentencias $(3,1 \%)$.

- Urología: 85 sentencias (3\%).

Respecto al resultado, 1.523 sentencias (54\%) fueron absolutorias, mientras que 1.294 (46\%) fueron condenatorias o con estimación parcial o total de la demanda.

En referencia a la cuantía media de la indemnización fijada en las sentencias condenatorias (o con estimación total o parcial de la demanda) esta fue de 83.457,5 $€$. Agrupadas por áreas afines, la cuantía media de la indemnización fijada fue $80.076 €$ para especialidades médicas, $86.587 €$ para especialidades quirúrgicas, $62.898 €$ en urgencias y $470.080 €$ para unidades de cuidados intensivos (UCI).

\section{Discusión}

En primer lugar, los autores quieren señalar que este trabajo amplía y profundiza una publicación anterior de esta Escuela de Medicina Legal que había estudiado un intervalo temporal más pequeño, recogía un menor número de casos y contemplaba menos variables ${ }^{1}$.

En segundo lugar, hay que mencionar las limitaciones de la fuente de datos utilizada en este estudio y su posible sesgo. Como se mencionó anteriormente, los datos sobre reclamaciones por responsabilidad profesional médica proceden de sentencias judiciales de segunda y tercera instancia. Quedan fuera de nuestros datos las reclamaciones extrajudiciales y las sentencias de las primeras instancias no recurridas. Esto hace que la extrapolación de nuestros datos al conjunto total de las reclamaciones de pacientes contra médicos deba hacerse con cautela. Esta peculiaridad en la procedencia de nuestros datos también origina la excesiva preponderancia de la jurisdicción civil sobre el resto. Esto se debe a que muchas reclamaciones contra los profesionales sanitarios comienzan en la jurisdicción penal, pero al ser desestimadas pasan en un gran porcentaje a la vía civil, y de ahí a instancias superiores, siendo en ese momento recogidas en nuestras fuentes de datos. Otro posible sesgo deriva de los criterios de selección de las sentencias. El hecho de haber considerado unos estrictos criterios de inclusión ha dejado fuera muchas sentencias «incompletas» que se refieren a la praxis médica. No obstante, pensamos que los que presentamos son los datos disponibles más representativos de lo que ocurre en España.

La distribución temporal de las sentencias incluidas en nuestra base de datos se corresponde con las del resto de series comparables. Todas contemplan un incremento de las reclamaciones legales de pacientes hasta el año $2010^{1-6}$.

En lo referente a las especialidades médicas más reclamadas, nuestros datos coinciden básicamente con el resto de datos disponibles para España ${ }^{1,2}$. El trabajo clásico del INSALUD (período 1995-2000) ${ }^{3}$ señala como las especialidades más reclamadas COT $(14,38 \%)$ y GO $(11,78 \%)$. El último informe disponible de la clausurada Oficina del Defensor del Paciente de la Comunidad de Madrid ${ }^{4}$ indica que las especialidades médicas más reclamadas fueron GO $(23,49 \%)$, seguida de COT $(10,17 \%)$. El trabajo de ArimanyManso et al. muestra las especialidades de GO, COT y cirugía general, como las que tuvieron más reclamaciones ${ }^{2}$. Vemos, así pues, que todas las series españolas coinciden con nuestros datos en cuanto a las 2 especialidades médicas más reclamadas. Respecto a los datos internacionales, las concordancias no son tan claras. El trabajo de referencia a nivel internacional es el publicado por Jena en el año $2011^{7}$, y señala como las especialidades médicas más reclamadas anestesiología y reanimación (en nuestra serie la novena en frecuencia), la práctica médica general, seguidas de la de GO y COT. No obstante, hay que ser muy cautos al comparar estudios realizados con metodologías tan diferentes.

Respecto a los datos de sentencias condenatorias obtenidas en nuestro estudio, en primer lugar debemos señalar que no hemos encontrado datos equiparables en España con los que comparar los nuestros, pero sí en las series internacionales. Nuestros datos señalan la existencia de un $46 \%$ de sentencias en las que se considera probada la existencia de algún tipo de mala praxis médica. La serie de Jena ${ }^{7}$, correspondiente a Estados Unidos, las reduce a un $22 \%$.

No existen apenas datos publicados sobre las cuantías medias de indemnizaciones a las que dan lugar las sentencias condenatorias en el ámbito español con las que comparar nuestros resultados ${ }^{1,2}$. En nuestra base de datos, la cuantía media de las indemnizaciones es de 83.457,5 $€$. Las indemnizaciones más cuantiosas son las correspondientes a las UCI (470.080 €), seguidas a mucha distancia por las especialidades quirúrgicas $(86.587 €$ ) y las especialidades médicas $(80.076 €)$. Debemos ser cautos al valorar los datos procedentes de las UCI debido a su escaso número. Según los datos de la serie de Arimany-Manso ${ }^{2}$, la media de indemnización fue de $66.636 €$, siendo GO la especialidad que afrontó la mayoría de las indemnizaciones y tuvo las mayores indemnizaciones medias. Llama la atención la diferencia con los datos de Estados Unidos ${ }^{7}$ en los que la indemnización media fue de $274.887 \$(210.956 €)$ y las especialidades con indemnizaciones más altas fueron pediatría, anatomía patológica y GO (que nosotros incluimos dentro en las especialidades quirúrgicas). En cualquier caso consideramos estos 
datos importantes a la hora de determinar la cobertura de los seguros profesionales de responsabilidad civil de cada una de las especialidades ${ }^{14,15}$.

\section{Conclusión}

El presente estudio facilita un mapa de situación sobre el número de sentencias emitidas, distribuida por especialidades médicas. Esto permitirá en el ámbito de la Medicina Legal, la propuesta de estrategias dirigida a la prevención de riesgos legales en las diferentes áreas profesionales. El conocimiento de las indemnizaciones medias puede ser un buen indicador para que las compañías de seguros ajusten sus coberturas por responsabilidad profesional.

\section{Conflicto de intereses}

Los autores declaran no tener ningún conflicto de intereses.

\section{Bibliografía}

1. Perea Pérez B, Santiago Sáez A, Labajo González ME, Albarrán Juan ME, Sánchez Sánchez JA. Análisis de las consecuencias médico-legales de las reclamaciones judiciales de pacientes. Estudio comparativo de los casos de traumatología, ginecología y obstetricia, anestesiología y reanimación y odontoestomatología. Trauma. 2009;4:264-8.

2. Arimany-Manso J, Gómez-Durán EL, Aubia-Marimon J. Las reclamaciones sobre responsabilidad profesional por especialidades en un escenario diferente al estadounidense. Gac Sanit. 2013;27:92-3.

3. Memoria Estadística INSALUD. Madrid: Ministerio de Sanidad y Consumo; 1999.
4. Defensor del Paciente de la Comunidad de Madrid. Memoria anual 2007. Madrid: CAM; 2008.

5. Health Resources and Services Administration. National practitioner data bank. Washington, DC: Department of Health and Human Services; 2010.

6. Kane CK. Policy research perspectives. Medical liability claim frequency: A 2007-2008 snapshot of physicians. Chicago: American Medical Association; 2010. p. 1-7.

7. Jena AB, Seabury S, Lakdawalla D, Chandra A. Malpractice risk according to physician specialty. N Engl J Med. 2011;365:629-36.

8. Mello MM, Studdert DM, Brennan TA. The new medical malpractice crisis. N Engl J Med. 2003;348:2281-4. Erratum in: N Engl J Med 2003;349:1010.

9. Medallo J, Pujol A, Arimany J. Aspectos médico-legales de la responsabilidad profesional médica. Med Clin (Barc). 2006;126:152-6.

10. Studdert DM, Mello MM, Gawande AA, Gandhi TK, Kachalia A, Yoon C, et al. Claims, errors, and compensation payments in medical malpractice litigation. $\mathrm{N}$ Engl J Med. 2006;354:2024-33.

11. Chandra A, Nundy S, Seabury SA. The growth of physician medical malpractice payments: evidence from the National Practitioner Data Bank. Health Aff (Millwood). 2005; Suppl Web Exclusivesp:W5-240-W5-249.

12. Baicker K, Fisher ES, Chandra A. Malpractice liability costs and the practice of medicine in the Medicare program. Health Aff (Millwood). 2007;26:841-52.

13. Mello MM, Chandra A, Gawande AA, Studdert DM. National costs of the medical liability system. Health Aff (Millwood). 2010;29:1569-77.

14. Perea-Pérez B, Santiago-Sáez A, Labajo-González E, AlbarránJuan ME, Sánchez-Sánchez JA. Consecuencias de las reclamaciones judiciales sobre los médicos afectados. Rev Clin Esp. 2011;211:17-22.

15. Bruguera M, Arimany J, Bruguera R, Barbería E, Ferrer F, Sala J, et al. Rev Clin Esp. 2012;212:198-205. 